\title{
A test of Rescorla and Wagner's (1972) prediction of nonlinear effects in contingency learning
}

\author{
Joaquín Morís • Susana Carnero • Ignacio Loy
}

Published online: 28 March 2012

(C) Psychonomic Society, Inc. 2012

\begin{abstract}
According to most theories, in a simple contingency learning situation, excitatory learning occurs when the probability of the unconditioned stimulus in the presence of the conditioned stimulus $\left(\mathrm{p}_{1}\right)$ is higher than the probability of the unconditioned stimulus in the absence of the conditioned stimulus $\left(\mathrm{p}_{2}\right)$. In Rescorla and Wagner's (1972) model, this prediction varies, depending on the parameters used. In the following experiments, we evaluated whether the difference between $p_{1}$ and $p_{2}$ that is required to produce excitatory conditioning is the same, independent of the specific value of $\mathrm{p}_{1}$, or whether this difference varies proportionally to $\mathrm{p}_{1}$ 's value. To do so, an appetitive procedure of Pavlovian conditioning with rats was used. In four experiments, we compared different levels of contingency (low, medium and high) and found that the difference between $\mathrm{p}_{1}$ and $\mathrm{p}_{2}$ that is required to produce excitatory conditioning increases when the value of $\mathrm{p}_{1}$ is higher. The possibility of analyzing contingency learning as a discrimination between $\mathrm{p}_{1}$ and $\mathrm{p}_{2}$ is also discussed.
\end{abstract}

Keywords Contingency learning · Associative learning · Excitatory conditioning $\cdot$ Psychophysics .

Discrimination · Rescorla and Wagner's (1972) model

Electronic supplementary material The online version of this article (doi:10.3758/s13420-012-0070-x) contains supplementary material, which is available to authorized users.

J. Morís $(\bowtie)$

Departamento de Psicología Básica, Universidad de Málaga,

Málaga 29071, Spain

e-mail: jmoris@uma.es

S. Carnero $\cdot$ I. Loy

University of Oviedo,

Oviedo, Spain

\section{Introduction}

In many situations, organisms need to learn about the relationships between cues and their consequences. In the simplest case, a cue is paired with a significant outcome and can be used to predict the occurrence of that outcome. In nonhuman animal experiments, the cue is a conditioned stimulus (CS), and its relevant consequence is the unconditioned stimulus (US). If both of them were binary, there would be four possible presentations of the stimuli. At a given trial, the CS and the US can appear together, the CS can appear alone, the US can appear alone, or neither of them may be present. We call the frequencies of each of these possible combinations $a, b, c$, and $d$ (see Table 1, e.g., Crump, Hannah, Allan, \& Hord, 2007). Using the frequencies of each type of trial, the conditional probabilities of the US given the CS $\left(\mathrm{p}_{1}\right)$, and of the US given the absence of the CS $\left(\mathrm{p}_{2}\right)$, can be calculated. The value of $\mathrm{p}_{1}$ is $a /(a+b)$, and the value of $\mathrm{p}_{2}$ is $c /(c+d)$.

Learning the relationship between a CS and a US is the basis of the conditioning, and many theories and models have been proposed to explain such contingency learning. In 1968, Rescorla reported a series of conditioned suppression experiments in which the difference between $\mathrm{p}_{1}$ and $\mathrm{p}_{2}$ determined whether learning was observed or not. According to Rescorla, a CS will produce an excitatory conditioned response if it is an informative predictor of an increase in the probability of the appearance of the US.

Rescorla and Wagner's (1972) model had, among others things, the objective of explaining these results. The model is usually expressed using two formulas, $\Delta V=\alpha \beta_{1}$ $\left(\lambda_{1}-\sum V_{X_{i}}\right)$ and $\Delta V=\alpha \beta_{2}\left(\lambda_{2}-\sum V_{X_{i}}\right)$. Parameters $\alpha$ and $\beta$ are associated to the CS and US salience, respectively. The parenthesis formulation represents the difference between the asymptotic level of associative strength that a 
Table 1 Trial types in contingency learning

\begin{tabular}{lll}
\hline & $C S$ & $\overline{C S}$ \\
\hline $\mathrm{US}$ & $a$ & $c$ \\
$\overline{U S}$ & $b$ & $d$ \\
\hline
\end{tabular}

$C S$ and $U S$ indicate the presence of the conditioned and unconditioned stimuli, respectively, and $\overline{C S}$ and $\overline{U S}$ indicate their absence. The frequencies of each trial type are represented with the letters $a, b, c$, and $d$

US is able to support $(\lambda)$ and the total associative strength that is governed by the stimuli on the present trial $\left(V_{X i}\right)$. The first formula is applied when the US is presented in that trial, the second when it is absent. Parameters $\beta_{1}$ and $\beta_{2}$ represent, respectively, the salience of the US and of its absence. In a similar fashion, $\lambda_{1}$ would be the maximum associative strength supported by the US, and $\lambda_{2}$ by the absence of the US.

Rescorla and Wagner (1972) ran several simulations of their model, showing that, through trial-by-trial learning, it can predict phenomena such as contingency learning and blocking (e.g., Kamin, 1969). In the years that followed, evidence of new phenomena predicted by the model, such as superconditioning (e.g., Rescorla, 2004) and overexpectation (e.g., Lattal \& Nakajima, 1998), was found. Rescorla and Wagner's model, and other models that share with it many features (e.g. Mackintosh, 1975; Pearce \& Hall, 1980; Van Hamme \& Wasserman, 1994) had been found relevant to not only animal associative learning but also to fields such as artificial intelligence, neuroscience, or categorization (e.g., Gluck, 1991; Luque, López, Marco-Pallares, Cámara, \& Rodríguez-Fornells, in press; Schultz, 2002; Soto \& Wasserman, 2010).

Many other models and rules were developed to study contingency learning. One of the most used is $\Delta \mathrm{p}$ (Allan, 1980; Jenkins \& Ward, 1965). It is a normative model of covariation between events. In this case, the events would be the CS and the US. It states that $\Delta \mathrm{p}=\mathrm{p}_{1}-\mathrm{p}_{2}$. Positive values of $\Delta p$ indicate an excitatory relationship between the $\mathrm{CS}$ and the US, whereas negative values indicate an inhibitory relationship. A value of 0 indicates that they are independent; that is, the presence or the absence of the CS gives no information about the occurrence of the US. As can be seen, it is equivalent to the rule proposed by Rescorla (1968). Following $\Delta p$, other rules were developed (see Allan, 1993, for a review in humans, and Hammond \& Paynter, 1983, for nonhumans). Chapman and Robbins (1990) showed that the asymptotic predictions of Rescorla and Wagner's (1972) model for a partially reinforced CS were equivalent to those of $\Delta p$ if two assumptions were made. The first one was to assume that a contextual stimulus was present in every trial. The second was that the salience of the presence and absence of the US was equal. That is, $\beta_{1}$ and $\beta_{2}$ should have the same value.

The assumption about the values of $\beta_{1}$ and $\beta_{2}$ is theoretically relevant, because using different values can change the predictions of the model in some circumstances. An example of this comes from the relative validity phenomenon (Wagner, Logan, Haberlandt, \& Price, 1968). Wagner et al. (1968) reinforced two compounds (AX and BX) differentially in two groups. In the first group, the correlated group, AX was reinforced $100 \%$ of the trials, whereas BX was never reinforced. In the second group, the uncorrelated group, both compounds were reinforced on $50 \%$ of the trials. In both groups, $\mathrm{X}$ was reinforced half of the times it was presented, and according to $\Delta p$, it should show the same level of conditioning. Contrary to this prediction, the uncorrelated group showed a higher level of X conditioning than the correlated group. This result is predicted by Rescorla and Wagner's (1972) model only if $\beta_{1}$ is higher than $\beta_{2}$. When $\beta_{1}$ and $\beta_{2}$ have the same value, the model predicts that $X$ should have the same associative strength in both the correlated and the uncorrelated groups. On the other hand, if $\beta_{1}$ is higher than $\beta_{2}$, it predicts correctly that $\mathrm{X}$ in the uncorrelated group should have more associative strength than in the correlated group (see Murphy, Baker, \& Fouquet, 2001, for an extensive discussion). In their original work, Rescorla and Wagner used values of 0.2 for $\beta_{1}$ and 0.1 for $\beta_{2}$, which would lead to a correct prediction. These same values are very often used when this model is used in animal learning literature (e.g., Miller, Barnet, \& Grahame, 1995).

Rescorla (1968), Rescorla and Wagner's (1972) model, when $\beta_{1}$ and $\beta_{2}$ have the same value, and $\Delta \mathrm{p}$ all predict the same result: If a difference between $p_{1}$ and $p_{2}$ were enough to provoke excitatory conditioning, this should happen independently of the specific values of $\mathrm{p}_{1}$ and $\mathrm{p}_{2}$. For example, a group of rats trained with $\mathrm{p}_{1}=0.1$ and $\mathrm{p}_{2}=0$ would have a $\Delta p=0.1$, and the CS should have an asymptotic associative strength of 0.1 . The same would be true for a group with $\mathrm{p}_{1}=$ $0.9, \mathrm{p}_{2}=0.8$, or a third that had $\mathrm{p}_{1}=0.5$ and $\mathrm{p}_{2}=0.4$. If excitatory conditioning were found in one of them, it should be found in all three.

But, what would be the prediction if $\beta_{1}$ were higher than $\beta_{2}$ ? In this situation, from a psychological point of view, trials in which the US is present would be more salient and relevant than those in which the US is absent. From the point of view of the model, because of the difference between $\beta_{1}$ and $\beta_{2}$, in those trials in which the US is presented, the change of the associative strength would tend to be higher than in those in which the US does not appear. As the proportion of trials in which the US is presented changes, this might lead to a change in the predictions.

To answer this question, a simulation with Rescorla and Wagner's (1972) model was run (for details of the simulations, see Appendix 1), using values of 0.2 for $\beta_{1}$ and 0.1 for 
$\beta_{2}$. The results of the simulation for the CS and the context are presented in Fig. 1. The pattern of predictions for the conditioning of the CS with these parameters is very different to that mentioned before (context conditioning will be discussed below). If $\beta_{1}$ is higher than $\beta_{2}$, then the same difference of $p_{1}-p_{2}$ leads to different asymptotic associative strengths of the CS, depending on the specific values of $\mathrm{p}_{1}$ and $\mathrm{p}_{2}$. A group trained with $\mathrm{p}_{1}=0.1$ and $\mathrm{p}_{2}=0$ is now predicted to have a higher asymptotic associative strength than those trained with $\mathrm{p}_{1}=0.9$ and $\mathrm{p}_{2}=0.8$, or $\mathrm{p}_{1}=0.5$ and $\mathrm{p}_{2}=0.4$. When $\mathrm{p}_{1}$ is $0.9, \mathrm{p}_{2}$ should be 0.6 to predict the level of conditioning obtained in the case of $\mathrm{p}_{1}=0.1$ and $\mathrm{p}_{2}=0$. When $\mathrm{p}_{1}$ is 0.5 , a $\mathrm{p}_{2}$ of 0.3 would be required to obtain an equivalent level of conditioning. Therefore, with these parameters, Rescorla and Wagner's model predicts that as the value of $p_{1}$ increases, the difference between $p_{1}$ and $p_{2}$ that is necessary to produce excitatory learning will increase. It should be noted that this is a robust prediction that occurs whenever $\beta_{1}$ is higher than $\beta_{2}$, independent of their specific values and of the values of the salience of the CS
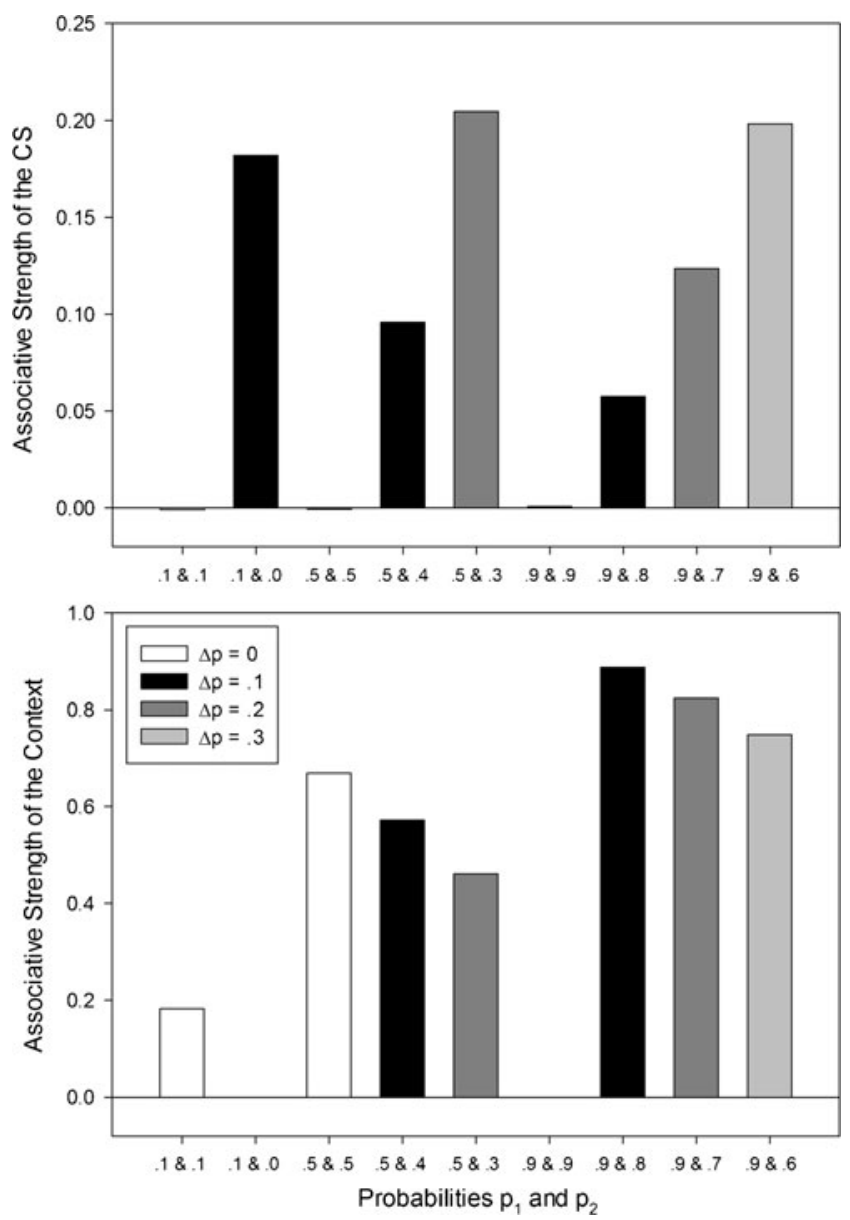

Fig. 1 Predictions of asymptotic associative strength of the CS (top panel) and the context (bottom panel) by the Rescorla and Wagner's (1972) model when $\beta_{1}=0.2$ and $\beta_{2}=0.1$. The color of the bars represents the value of $\Delta \mathrm{p}$ for that combination of $\mathrm{p}_{1}$ and $\mathrm{p}_{2}$ and of the context (see the Supplemental Data for additional simulations).

The aim of the present study was to test that prediction using an appetitive procedure in rats. The objective of the series of experiments presented was to see whether the difference between $p_{1}$ and $p_{2}$ needed to produce an increase in the conditioned responding to the CS is the same for all the values of $\mathrm{p}_{1}$, or whether this difference becomes higher as the value of $p_{1}$ increases. In Experiment $1 a$, we tested whether a group with contingency $\mathrm{p}_{1}=0.1$ and $\mathrm{p}_{2}=0$ has a higher rate of responding to the CS than a control group receiving a training in which $\mathrm{p}_{1}=0.1$ and $\mathrm{p}_{2}=0.1$. In Experiment 1b, we explored whether that same difference would be enough to produce an increase in the conditioned response when $p_{1}$ and $p_{2}$ have much higher values, $p_{1}=0.9$ and $\mathrm{p}_{2}=0.8$ in the case of the experimental group, and $\mathrm{p}_{1}=$ 0.9 and $\mathrm{p}_{2}=0.9$ for its control group. In Experiment 2, a difference between $p_{1}$ and $p_{2}$ that was high enough to produce conditioning when $\mathrm{p}_{1}$ was 0.9 was investigated. In Experiment 3, the effect was tested at a medium contingency level not used before, $p_{1}=0.5$. Finally, in Experiment 4, we replicated the findings of Experiments $1 \mathrm{a}$ and $1 \mathrm{~b}$ in the same experiment, with a different procedure that allowed better measuring of context conditioning (see Table 2 for all the frequencies employed in the four experiments).

\section{Experiment 1}

\section{Experiment 1a}

The aim of this Experiment 1 was to test the extent to which a magazine training procedure in rats was able to show contingency learning at a low contingency level. To do this, in the experimental group, $\mathrm{p}_{1}$ had a value of 0.1 , and $\mathrm{p}_{2}$ of 0 . Note that for all of the comparisons in this experiment, and in the following ones, a group in which the value of $\mathrm{p}_{2}$ is the same as that of $p_{1}$ is used as the control. In previous studies, researchers have shown that control groups can show some change of performance (e.g., Kirkpatrick \& Church, 2004; see Papini \& Bitterman, 1990, for a review). In our procedure, excitatory conditioning will be understood as a differential increase in responses during the presence of the CS in the experimental groups as compared with the control group (using an elevation ratio, which will be explained later in more detail, as a dependent measure). Thus, the presentation of a salient CS might increase the arousal of rats so that the CS would have an elevation ratio over 0.5 , even although no learning has been acquired. Such problems are obviated by making a comparison with the control group described. Baker, Murphy, Vallee-Tourangeau, and Mehta (2001) discussed extensively the advantages of using this kind of control instead of normative ones. 
Table 2 Probabilities and frequencies of reinforcement

\begin{tabular}{|c|c|c|c|c|c|}
\hline Experiment & Group & $\mathrm{p}_{1}$ & Reinforced and total CS trials & $\mathrm{p}_{2}$ & Reinforced and total non-CS trials \\
\hline \multirow[t]{2}{*}{$1 \mathrm{a}$} & Control: $0.1 \& 0.1$ & 0.1 & $1 / 10$ & 0.1 & $8 / 80$ \\
\hline & Experimental: $0.1 \& 0.0$ & 0.1 & $1 / 10$ & 0.0 & $0 / 80$ \\
\hline \multirow[t]{2}{*}{$1 \mathrm{~b}$} & Control: $0.9 \& 0.9$ & 0.9 & $9 / 10$ & 0.9 & $72 / 80$ \\
\hline & Experimental: $0.9 \& 0.8$ & 0.9 & $9 / 10$ & 0.8 & $64 / 80$ \\
\hline \multirow[t]{4}{*}{2} & Control: $0.9 \& 0.9$ & 0.9 & $9 / 10$ & 0.9 & $72 / 80$ \\
\hline & Experimental 1: $0.9 \& 0.75$ & 0.9 & $9 / 10$ & 0.75 & $60 / 80$ \\
\hline & Experimental 2: $0.9 \& 0.7$ & 0.9 & $9 / 10$ & 0.7 & $56 / 80$ \\
\hline & Experimental 3: $0.9 \& 0.6$ & 0.9 & $9 / 10$ & 0.6 & $48 / 80$ \\
\hline \multirow[t]{3}{*}{3} & Control: $0.5 \& 0.5$ & 0.5 & $5 / 10$ & 0.5 & $40 / 80$ \\
\hline & Experimental 1: $0.5 \& 0.4$ & 0.5 & $5 / 10$ & 0.4 & $32 / 80$ \\
\hline & Experimental 2: $0.5 \& 0.3$ & 0.5 & $5 / 10$ & 0.3 & $24 / 80$ \\
\hline \multirow[t]{4}{*}{4} & Control 1: $0.1 \& 0.1$ & 0.1 & $3 / 30$ & 0.1 & $3 / 30$ \\
\hline & Experimental 1: $0.1 \& 0.0$ & 0.1 & $3 / 30$ & 0.0 & $0 / 30$ \\
\hline & Control 2: $0.9 \& 0.9$ & 0.9 & $27 / 30$ & 0.9 & $27 / 30$ \\
\hline & Experimental 2: $0.9 \& 0.8$ & 0.9 & $27 / 30$ & 0.8 & $24 / 30$ \\
\hline
\end{tabular}

Frequencies of reinforcement delivered in each session are summarized in Table 2. For every group, the probabilities $\mathrm{p}_{1}$ and $\mathrm{p}_{2}$ and Reinforced and total CS trials Reinforced and total non-CS trials are shown

In Experiment 1a, the control group received 0.1 and 0.1 as $\mathrm{p}_{1}$ and $\mathrm{p}_{2}$, respectively. If experimental animals are able to show significantly more responding to the CS than are control animals, at low levels of contingency, then a difference between $\mathrm{p}_{1}$ and $\mathrm{p}_{2}$ of 0.1 would be enough to produce an increase in responding, and the paradigm with the parameters employed would have shown to be suitable for the investigation of other levels of contingency.

\section{Method}

Subjects Subjects were 16 experimentally naive male Wistar rats that were about 120 days old and that had an ad libitum weight of 502 (range 397-580 g.). They were housed in cages, each of which containing four rats. They had free access to water. One hour after each session, they were fed to maintain them at $80 \%$ of their ad libitum weight. Each day, there were 12 hours of light, beginning at 8 a.m. The experiment was run during this light phase.

Apparatus The apparatus consisted of eight identical operant chambers measuring $24 \times 29 \times 38 \mathrm{~cm}$. The front and the back walls were made of aluminium, the side walls and the ceiling of transparent methacrylate, and the floor was composed of $0.4 \mathrm{~cm}$ stainless steel rods, spaced $1 \mathrm{~cm}$ apart. The center end of the front wall contained a recessed food magazine measuring $6 \times 3.5 \times 6 \mathrm{~cm}$ that was located $0.5 \mathrm{~cm}$ above the floor. Each chamber was enclosed in a sound- and light-attenuating shell. A speaker that produced a $600-\mathrm{Hz}$ and $76-\mathrm{dB}$ SPL tone, which was used as the CS, was mounted on the front wall,
$8 \mathrm{~cm}$ over the food magazine. A fan included in the shell produced a background noise of $62 \mathrm{~dB}$ SPL. A pellet dispenser mounted behind the food magazine dispensed $45 \mathrm{mg}$ pellets (type Test Diet-MLab Rodent Tablet) through a tube connecting the magazine and the dispenser. Photocells located in the magazine recorded entries into the food magazine in both presence and absence of the CS.

Procedure Rats were randomly assigned to two groups of eight subjects each and then received two days of magazine training and 22 sessions of conditioning.

Magazine training On Days 1 and 2, subjects received a 20-min session of magazine training during which pellets were delivered according to a variable-time 120 -s schedule. Four pellets were placed in the magazine before the beginning of these sessions.

Conditioning Conditioning began on Day 3 and continued through Day 24 (a total of 22 sessions). Conditioning sessions were designed to be an appetitive procedure equivalent to that used by Rescorla (1968). Each session lasted $960 \mathrm{~s}$. The first and last $30 \mathrm{~s}$ had no event scheduled. The remaining $900 \mathrm{~s}$ were divided into 10 -s. bins. In 10 of these bins, the tone used as the CS was presented for the duration of the bin. In the remaining time bins, the CS was absent. The mean interval between CS trials was $90 \mathrm{~s}$, the minimum was $70 \mathrm{~s}$, and the maximum was $110 \mathrm{~s}$. (Bueno \& Álvarez, 2001; Holland, 2000). A food pellet, the US, could be delivered during the last second of each time bin. Whenever the CS was present, the probability of this happening was 
$\mathrm{p}_{1}$, as set in the experiment, and in those bins in which the CS was absent, the probability was $\mathrm{p}_{2}$ (Table 2 shows the exact number of trials reinforced and nonreinforced). In Experiment $1 \mathrm{a}$ these values were $\mathrm{p}_{1}=0.1$ for both the Experimental group and the Control group. The value of $\mathrm{p}_{2}$ for the Experimental group was 0, and for Control group, 0.1. This means that for the Experimental group, on average, a US would appear once every $10 \mathrm{CS}$ trials, but no USs would be presented in absence of the CS. On the other hand, the Control group would receive food, on average, once every $10 \mathrm{CS}$ trials and also once every 10 no-CS trials.

The conditioned response controlled by the CS was computed as an elevation ratio. The value of this ratio was the number of magazine entries during the presentation of the CS divided by the sum of this number, and the number of magazine entries in the previous $10 \mathrm{~s}$. Therefore, higher values of the ratio reflect more excitatory conditioning of the CS. To ensure that the effects found were asymptotic, only the last eight sessions were analyzed.

Data analysis SPSS 14 was used to analyze the data. Sphericity was tested in every repeated measures ANOVA and, when needed, degrees of freedom were adjusted using the Greenhouse-Geisser correction. The level of significance used was $\alpha=0.05$.

\section{Results}

The mean elevation ratios for the two groups of Experiment $1 \mathrm{a}$ during the last eight sessions (from the fifteenth to twentysecond) are presented in Fig. 2. As can be seen in Fig. 2, animals in the Experimental group showed higher elevation

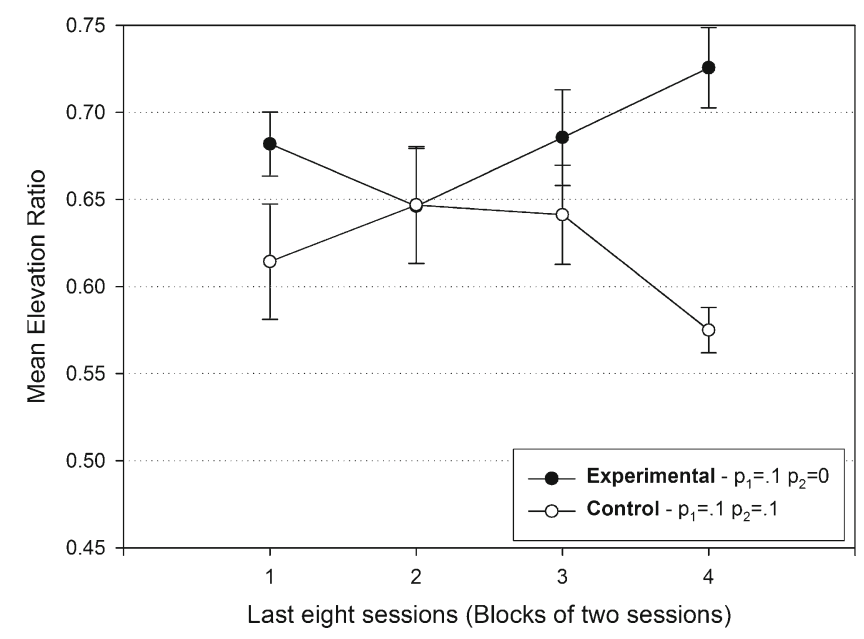

Fig. 2 Mean elevation ratio of the responses to the CS of the experimental and control groups of Experiment 1a in the last eight conditioning sessions. Error bars represent the standard errors of the means ratio scores than controls, especially in those blocks that represent sessions 19 to 22 (blocks 3 and 4).

A repeated measures ANOVA of the last eight sessions, using group and session as factors, showed a statistically significant effect of the factor group, $F(1,14)=5.74, p<.05$. There was no effect of session, $F(7,98)=0.60, p>.05$, or of interaction between both factors, $F(7,98)=2.00, p>.05$. The mean pre-CS responding of the Control group was 0.69 $(S D=1.14)$, and the mean of the Experimental group was 1.04 $(S D=1.97)$. Another ANOVA showed that there were no statistical differences in the levels of responding previous to the CS, $F(1,14)=1.62, p>.05$, indicating that groups did not differ in its CR to the context. A difference of 0.1 between $\mathrm{p}_{1}$ and $\mathrm{p}_{2}$ was enough to show more excitatory conditioning with low values of $\mathrm{p}_{1}$ than in a control group trained with no difference between the two probabilities.

\section{Experiment 1b}

In Experiment 1a, we showed that with the current procedure, a value of $\Delta \mathrm{p}=0.1$ is enough to produce excitatory conditioning when $\mathrm{p}_{1}=0.1$. If the difference between $\mathrm{p}_{1}$ and $\mathrm{p}_{2}$ that is needed to produce excitatory conditioning is absolute and constant for every value of $\mathrm{p}_{1}$, then a group with $\Delta \mathrm{p}=0.1$ having $\mathrm{p}_{1}=0.9$ and $\mathrm{p}_{2}=0.8$ should show a higher level of responding to the CS than a control group trained with $\mathrm{p}_{1}$ and $\mathrm{p}_{2}$ equal to 0.9 . On the other hand, according to Rescorla and Wagner's (1972) model with different values for $\beta_{1}$ and $\beta_{2}$, the difference between these two groups should be very small. To test this prediction, these two groups were used in Experiment $1 \mathrm{~b}$ as experimental group and control group, respectively.

\section{Method}

The experimental subjects were 16 naive male Wistar rats that at the beginning of the experiment were 120 days old and had an ad libitum weight of $499 \mathrm{~g}$ (range 432-561 g). All of the maintenance details were the same as in Experiment 1a. The procedure used was the same as in Experiment 1a, and only the contingencies of the groups were changed (see Table 2).

\section{Results}

The mean elevation ratios for the groups of Experiment $1 \mathrm{~b}$ are presented in Fig. 3. As can be seen in the figure, both groups showed similar levels of $\mathrm{CR}$ in the last eight sessions.

A repeated measures ANOVA of the last eight sessions showed no statistically significant effect of the group factor, $F(1,13)=0.01, p>.05$. There was a significant effect of 


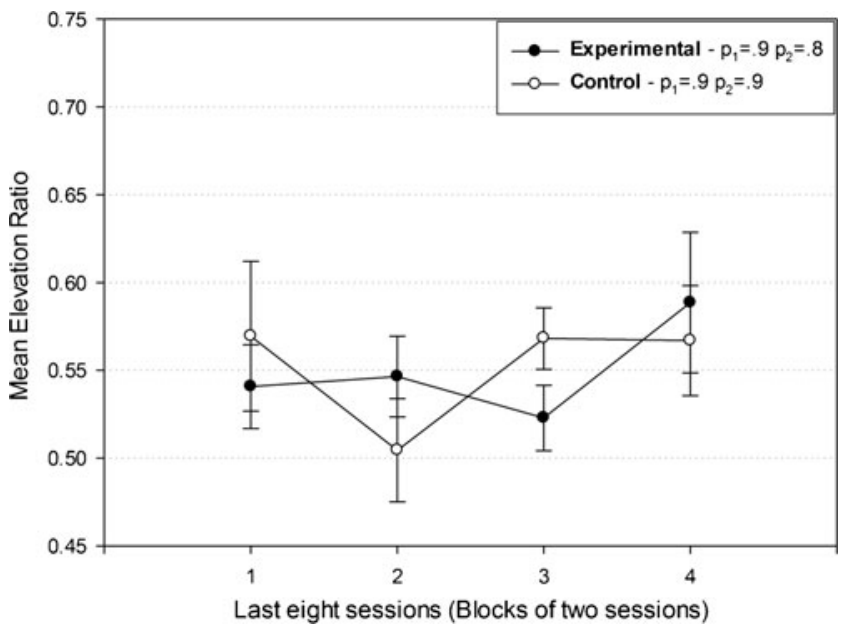

Fig. 3 Mean elevation ratio of the responses to the CS of the experimental and control groups of Experiment $1 \mathrm{~b}$ in the last eight conditioning sessions. Error bars represent the standard errors of the means

session, $F(2.5,32.6)=3.12, p<.05$ but no interaction between the factors, $F(2.5,32.6)=0.89, p>.05$. The mean pre-CS responding was $7.55(S D=4.91)$ in the Control group and $6.88(S D=4.60)$ in the Experimental group. No statistical differences between the groups were found in the levels of responding previous to the $\mathrm{CS}, F(1,13)=0.65, p>.05$.

The difference between the probabilities of the US in the presence and absence of the CS, which produced an increase in responding to the CS when $p_{1}$ value was low (Experiment 1a), was not enough to produce a similar effect when $p_{1}$ value was high (Experiment 1b).

\section{Experiment 2}

The difference between $p_{1}$ and $p_{2}$ that produced an increase in conditioned responding as compared with its control when $\mathrm{p}_{1}$ was 0.1 did not produce it when $\mathrm{p}_{1}$ had a value of 0.9. Experiment 2 was designed to determine a difference between $p_{1}$ and $p_{2}$ that was high enough to produce more excitatory conditioning than a control when $\mathrm{p}_{1}=0.9$. To do this, four groups were used: $a$ Control group $\left(\mathrm{p}_{1}=0.9\right.$ and $\mathrm{p}_{2}=$ 0.9 ) and three experimental groups that had increasing values of $\Delta \mathrm{p}$. They were Experimental $1\left(\mathrm{p}_{1}=0.9\right.$ and $\left.\mathrm{p}_{2}=0.75\right)$, Experimental $2\left(\mathrm{p}_{1}=0.9\right.$ and $\left.\mathrm{p}_{2}=0.7\right)$, and Experimental $3\left(\mathrm{p}_{1}=0.9\right.$ and $\left.\mathrm{p}_{2}=0.6\right)$. They had $\Delta \mathrm{p}$ values of, respectively, $0.15,0.2$, and 0.3 (see Table 2).

\section{Method}

The experimental subjects were 32 naive male Wistar rats that were 90 days old and had an ad libitum weight of $437 \mathrm{~g}$ (range 378-508 g) at the beginning of the experiment. All of the maintenance details were the same as in Experiments 1a and $1 \mathrm{~b}$. The procedure used was the same as in previous experiments, and only the contingencies of the groups were changed.

\section{Results}

The mean elevation ratios for the groups of Experiment 2 during the last eight sessions are presented in Fig. 4. It can be seen that group Experiment $3\left(\mathrm{p}_{1}=0.9\right.$ and $\left.\mathrm{p}_{2}=0.6\right)$ showed higher levels of CR than the other groups.

By conducting a repeated measures ANOVA of the last eight sessions, we found a statistically significant difference among the groups, $F(3,28)=3.71, p<.05$, a significant effect of the sessions, $F(4.8,135.7)=4.13, p<.05$, and a significant interaction between these factors, $F(14.5,135.7)=$ $2.43, p<.05$. A post hoc Tukey of the means of those last eight sessions test showed that only the difference between the Control group $\left(\mathrm{p}_{1}=0.9\right.$ and $\left.\mathrm{p}_{2}=0.9\right)$ and the Experimental group $3\left(\mathrm{p}_{1}=0.9\right.$ and $\left.\mathrm{p}_{2}=0.6\right)$ was statistically significant. The mean number of responses during the pre-CS period of the Control group and Experimental Groups 1, 2, and 3 was, respectively, $6.91(S D=3.34), 8.87(S D=4.44), 9.62$ $(S D=5.34)$, and $6.36(S D=3.16)$. No statistical differences were found in the levels of responding previous to the $\mathrm{CS}, F(3,28)=1.15, p>.05$.

Therefore, when $\mathrm{p}_{1}$ value was high, a difference between $\mathrm{p}_{1}$ and $\mathrm{p}_{2}$ of 0.3 was enough to produce an increase in conditioned responses to the CS, but differences of 0.2 and 0.15 were not.

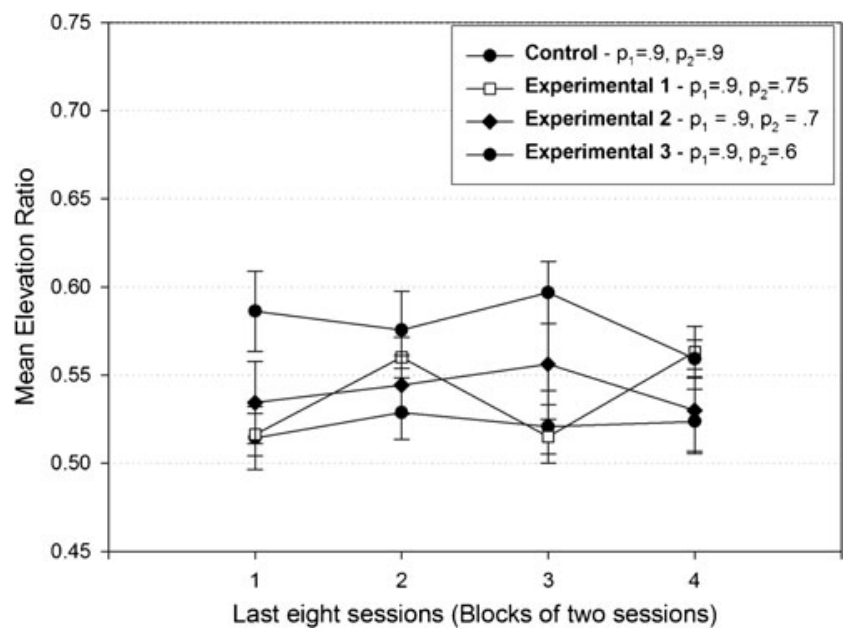

Fig. 4 Mean elevation ratio of the responses to the CS of the experimental and control groups of Experiment 2 in the last eight conditioning sessions. Error bars represent the standard errors of the means 


\section{Experiment 3}

In Experiment 3, a similar design to those of Experiments 1 and 2 was used, but this time the value of $p_{1}$ was medium, 0.5. The objective was to extend the findings of Experiments 1 and 2 to another level of $p_{1}$ value, and to test the predictions of Rescorla and Wagner's (1972) model also under those conditions. By doing so, a potential problem of Experiment 1a was solved. In Experiment 1, the experimental group $\mathrm{p}_{2}$ had a value of 0 . It might be argued that this value might have a special psychological significance and be more salient than other values in the continuum of probabilities. Then, the results of Experiment 1a might not be applicable to the whole contingency space, preventing a correct test of the discussed predictions of Rescorla and Wagner's model. In Experiment 3, therefore, we investigated the effects produced by $\Delta \mathrm{p}=0.1$, but in a procedure without values of $\mathrm{p}_{1}$ or $\mathrm{p}_{2}$, that might be of special relevance. One group was trained with $\Delta \mathrm{p}=0.1\left(\mathrm{p}_{1}=0.5\right.$ and $\left.\mathrm{p}_{2}=0.4\right)$; a second experimental group was trained with $\Delta \mathrm{p}=0.2\left(\mathrm{p}_{1}=0.5\right.$ and $\left.\mathrm{p}_{2}=0.3\right)$. Would these show excitatory conditioning as compared with a control group with $\Delta \mathrm{p}=0\left(\mathrm{p}_{1}=0.5\right.$ and $\mathrm{p}_{2}=$ 0.5)? (See Table 2).

\section{Method}

The experimental subjects were 24 naive male Wistar rats that, at the beginning of the experiment, were 90 days old and had an ad libitum weight of $430 \mathrm{~g}$ (range 363-506 g). All of the the maintenance details were the same as in previous experiments. The procedure used was the same as in Experiment 1a, and only the contingencies of the groups were changed.

\section{Results}

The mean elevation ratios of the groups of Experiment 3 during the last eight sessions of training are represented in Fig. 5. As can be seen in the figure, Experimental Group 2 $\left(p_{1}=0.5\right.$ and $\left.p_{2}=0.3\right)$ showed the highest level of CR.

A repeated measures ANOVA of the last eight sessions found statistically significant differences between the groups, $F(2,21)=25.77, p<.05$, a significant effect of the sessions, $F(7,147)=3.64, p<.05$, but no significant interaction between the factors, $F(14,147)=1.06, p>.05$. A post hoc Tukey test of the means of the eight sessions showed that the only difference that was statistically significant was between the Control group $\left(\mathrm{p}_{1}=0.5\right.$ and $\left.\mathrm{p}_{2}=0.5\right)$ and Experimental Group $2\left(p_{1}=0.5\right.$ and $\left.p_{2}=0.3\right)$. The mean number of responses during the pre-CS period of the Control group and Experimental Groups 1 and 2 was, respectively, $6.89(S D=4.19), 6.17(S D=3.89)$, and $4.72(S D=4.96)$. Another ANOVA found no statistical differences in responding previous to the $\mathrm{CS}, F(2,21)=2.24, p>.05$.

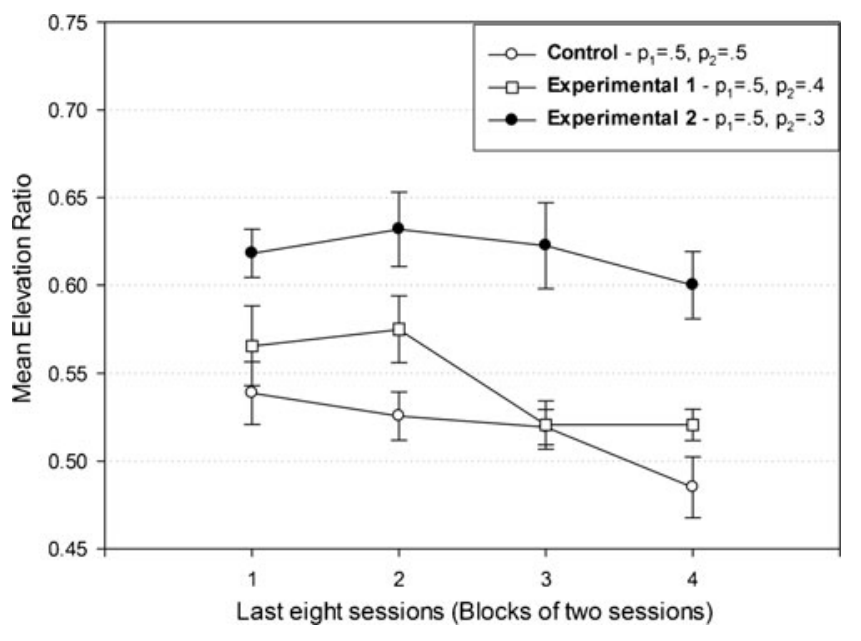

Fig. 5 Mean elevation ratio of the responses to the CS of the experimental and control groups of Experiment 3 in the last eight conditioning sessions. Error bars represent the standard errors of the means

\section{Experiment 4}

In Experiment $1 \mathrm{a}$, we showed that a difference of 0.1 between $\mathrm{p}_{1}$ and $\mathrm{p}_{2}$ was enough to produce an increase in conditioned responding to the CS when $p_{1}$ had a value of 0.1 . This difference did not produce the same effect in Experiment $1 \mathrm{~b}$, where $\mathrm{p}_{1}$ was 0.9 . In Experiment 4, we tried to replicate these two results in a single experiment. Also, the simulations presented in Fig. 1 show that Rescorla \& Wagner's (1972) model predicts that in each of the previous experiments, experimental and control groups should have differed in their level of context conditioning. For example, according to the predictions presented in Fig. 1, the associative strength of the context in a group with $\mathrm{p}_{1}=0.1$ and $\mathrm{p}_{2}=0$ should be zero, whereas one with $\mathrm{p}_{1}=0.1$ and $\mathrm{p}_{2}=$ 0.1 would be 0.2 . However, this was not found, although differences in responding to the CS were detected. This could have been because of the fact that the measure of context conditioning was less sensitive than that used to assess the CR. Responding to the CS was measured as an elevation ratio, to control a possible effect of context conditioning. This kind of measure allows additional advantages, because it also reduces the influence of additional factors such as general activity of the animal. Since the index of context responding was the number of responses while the CS was absent, it did not have this feature. Because of this, a different procedure was used in this experiment, in order to try to measure responding to the context in a situation equivalent to the CS. In this procedure, every trial was signaled with a discrete stimulus. Therefore, this stimulus appeared in all trials and functioned as a contextual cue that indicated that a trial was taking place. This stimulus could appear on some of the trials alone, followed or not by the US (these trials were used to calculate $\mathrm{p}_{2}$ ), whereas in 
others, it appeared with the CS, followed by the US or not (to calculate $\mathrm{p}_{1}$ ). As a result of this training, these types of trials defined the probabilities assigned to each group. Thanks to this change, an elevation ratio equivalent to that of the CS could be calculated for all of the trials, those that included the CS and those that did not, allowing a better comparison between these two situations. Therefore the basic analysis used in Experiments 1-3 was also applied to the modified procedure. Also, as can be seen in Fig. 6, the predictions of Rescorla and Wagner's model for this training are identical to those shown in Fig. 1 that were calculated for the training used in Experiments 1 to 3 (see Appendix 1).

Four groups were used; two of them were experimental groups with the same $\Delta p=0.1$, but with different values of $\mathrm{p}_{1}\left(\mathrm{p}_{1}=0.1\right.$ and $\left.\mathrm{p}_{1}=0.9\right)$. There were two equivalent control groups with the same $\mathrm{p}_{1}$ as the experimental groups and $\Delta \mathrm{p}=0$.
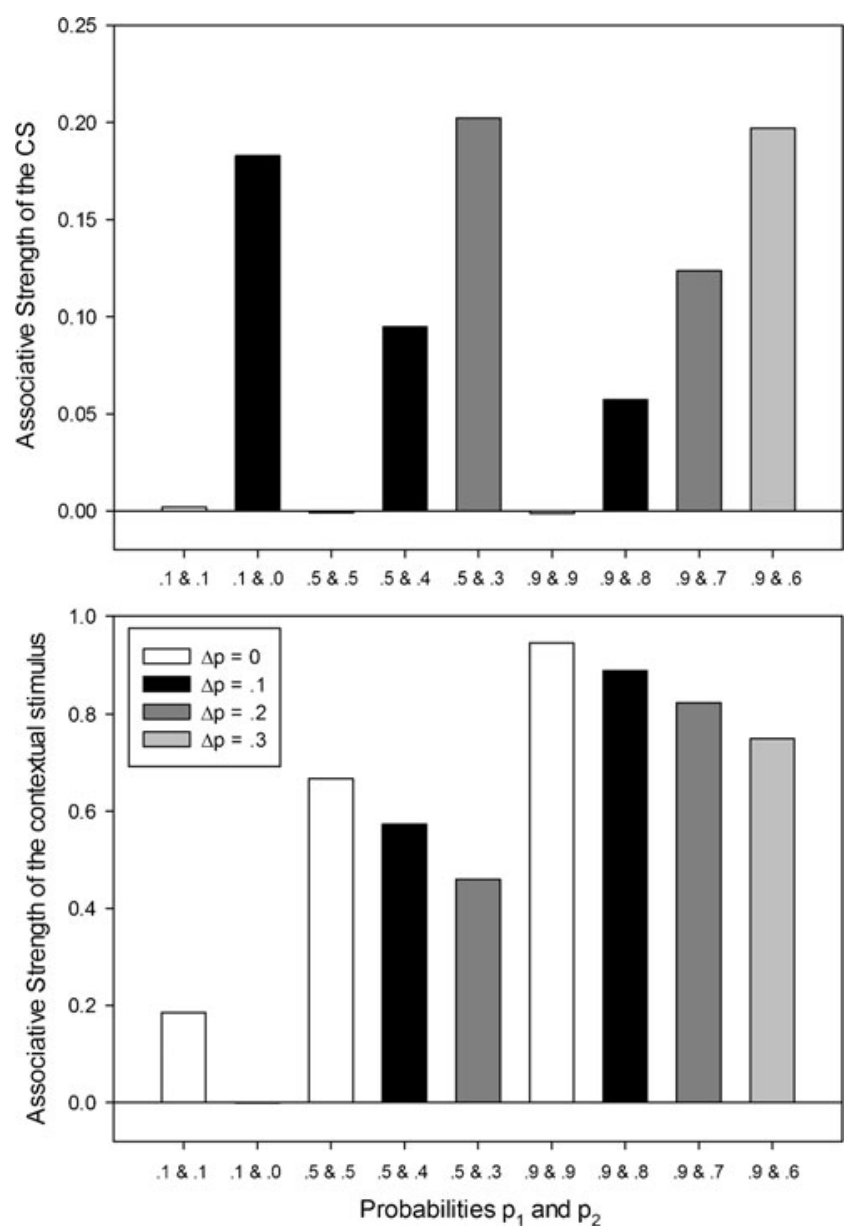

Fig. 6 Predictions of asymptotic associative strength of the CS (top panel) and the contextual stimulus (bottom panel) by Rescorla and Wagner's (1972) model for a contingency training in which a contextual cue is used to mark each trial. The value of the parameters used were $\beta_{1}=0.2$ and $\beta_{2}=0.1$. The color of the bars represents the value of $\Delta p$ for that combination of $p_{1}$ and $p_{2}$ (see Appendix 1 for more details)

\section{Method}

Subjects Subjects were 32 experimentally naive male Wistar rats about 120 days old that had an ad libitum weight of $383 \mathrm{~g}$ (range $347-411 \mathrm{~g}$ ). All of the maintenance details were the same as in previous experiments.

Apparatus The apparatus was the same as before, except for a new speaker that was added in Skinner boxes. It generated a new auditory stimulus: a 3,000-Hz and $82-\mathrm{dB}$ SPL intermittent click that lasted for $10 \mathrm{~s}$. This stimulus was used as a CS.

Procedure Rats were randomly assigned to four groups of eight subjects each and then received 2 days of magazine training and 16 sessions of conditioning. The procedure used was similar to that employed by Murphy and Baker (2004). Each session was composed of trials in which only a 10 -s tone was presented (no-CS trials) and trials in which the tone and a click, also $10 \mathrm{~s}$ long, were presented simultaneously (CS trials). Therefore, the click was used as a CS, and the tone served as a discriminative stimulus that indicated that a trial was taking place. This ensured that CS and no-CS trials were signaled in the same way.

Conditioning Conditioning began on Day 3 and continued until Day 18. Each session lasted $60 \mathrm{~min}$. In each session, 60 trials were presented, 30 with the presentation of the tone alone (non-CS trials), whereas in the other 30 , the tone and a click were presented (CS trials). The mean interval between trials was $60 \mathrm{~s}$, and the order of the trials was random. A food pellet, the US, could be delivered at the end of the trial. Each trial had a probability of reinforcement determined by the values of $p_{1}$ and $p_{2}$ of the group (Table 2 shows the exact number of reinforced and no reinforced trials). Four groups were established: Control Group $1\left(\mathrm{p}_{1}=0.1\right.$ and $\left.\mathrm{p}_{2}=0.1\right)$, in which both probabilities were the same and fixed in a low value; Experimental Group $1\left(\mathrm{p}_{1}=0.1\right.$ and $\left.\mathrm{p}_{2}=0.0\right)$, which was trained with a $\Delta \mathrm{p}=0.1$ and a low $\mathrm{p}_{1}$ value; Control Group $2\left(\mathrm{p}_{1}=0.9\right.$ and $\left.\mathrm{p}_{2}=0.9\right)$, which worked as control with an equal value of probabilities fixed in a high value; and, finally, Experimental Group $2\left(\mathrm{p}_{1}=0.9\right.$ and $\left.\mathrm{p}_{2}=0.8\right)$, which was a group trained with a $\Delta p=0.1$ and a high value of $\mathrm{p}_{1}$. In each session, the click was conditioned according to the probabilities stated. In Control Group $1\left(\mathrm{p}_{1}=0.1\right.$ and $\left.\mathrm{p}_{2}=0.1\right), 10 \%$ of tone and click $\left(\mathrm{p}_{1}\right)$ and tone-alone trials $\left(\mathrm{p}_{2}\right)$ were reinforced. Experimental Group $1\left(\mathrm{p}_{1}=0.1\right.$ and $\left.\mathrm{p}_{2}=0.0\right)$ received a food pellet on $10 \%$ of the 30 tone and click trials $\left(p_{1}\right)$, but none of the 30 tone-alone trials was followed by food $\left(\mathrm{p}_{2}\right)$. In Control Group $2\left(\mathrm{p}_{1}=0.9\right.$ and $\mathrm{p}_{2}=$ 0.9 ), $90 \%$ of both types of trials was reinforced. Finally, $90 \%$ of tone and click trials and $80 \%$ of the tone trials were reinforced in Experimental Group $2\left(\mathrm{p}_{1}=0.9\right.$ and $\left.\mathrm{p}_{2}=0.8\right)$. 
This method generated faster conditioning than that used in previous experiments, which is why the number of sessions was reduced as compared with those in previous experiments.

Data analysis Elevation ratios, similar to those explained before, were used for the analysis. The elevation ratio of the non-CS trials, in which the tone was presented alone $\left(\mathrm{ER}_{\text {tone }}\right)$, and of the CS trials, in which both the tone and the click were presented ( $\left.\mathrm{ER}_{\text {click }+ \text { tone }}\right)$, were calculated using the magazine entries recorded during the presentation of the stimuli and those registered in a previous period of the same duration, $10 \mathrm{~s}$. The dependent variable employed to analyze the conditioning data was the difference of elevation ratios of the two types of trials $\left(\mathrm{Dif}_{\mathrm{ER}}=\mathrm{ER}_{\text {click+tone }}-\mathrm{ER}_{\text {tone }}\right)$. By doing so, the conditioning of the CS was estimated. The final six sessions were analyzed, to avoid possible preasymptotic effects.

\section{Results}

In Fig. 7, we present the results of the last six conditioning sessions (collapsed in blocks of two) and show that Experimental Group $1\left(\mathrm{p}_{1}=0.1\right.$ and $\left.\mathrm{p}_{2}=0.0\right)$ had the highest asymptotic level of conditioned responses. To analyze the data, a repeated measures ANOVA was used. It had a within-subjects factor, session, and a between-subjects factor, group. No interaction effect between session and group, or a main session effect was found, $F<1$. However, the main effect of group was significant, $F(3,28)=18.897, p<$ .05 . A Tukey post hoc analysis showed significant differences in their CR between Experimental Group $1\left(\mathrm{p}_{1}=0.1\right.$ and $\left.\mathrm{p}_{2}=0.0\right)$ and the other three groups. No other difference was statistically significant.

To test for a possible difference in context conditioning, elevation ratios on CS and no-CS trials were analyzed separately. The results are presented in Fig. 8. Experimental Group 1 showed a higher ER on the CS trials, whereas the four groups showed a similar level of conditioning on no-CS trials. Another ANOVA was run, now with two withinsubjects factors, session and trial Type (CS vs. no CS). There was a significant interaction between trial type and group factors, $F(3,28)=23.57, p<.05$. Additional tests showed that there was no significant difference among groups in mean elevation ratio on no-CS trials $(F<1)$, but a significant difference was present in CS trials, $F(3,28)=$ $3.04, p<.05$. A Tukey test showed that this result was due to differences was between Experimental Group $1\left(\mathrm{p}_{1}=0.1\right.$ and $\left.\mathrm{p}_{2}=0.0\right)$ and the other three groups.

These results replicated those of Experiments $1 \mathrm{a}$ and $1 \mathrm{~b}$. A difference of $\Delta p$ of 0.1 was enough to produce an increase in responding to the CS in a group with a low value

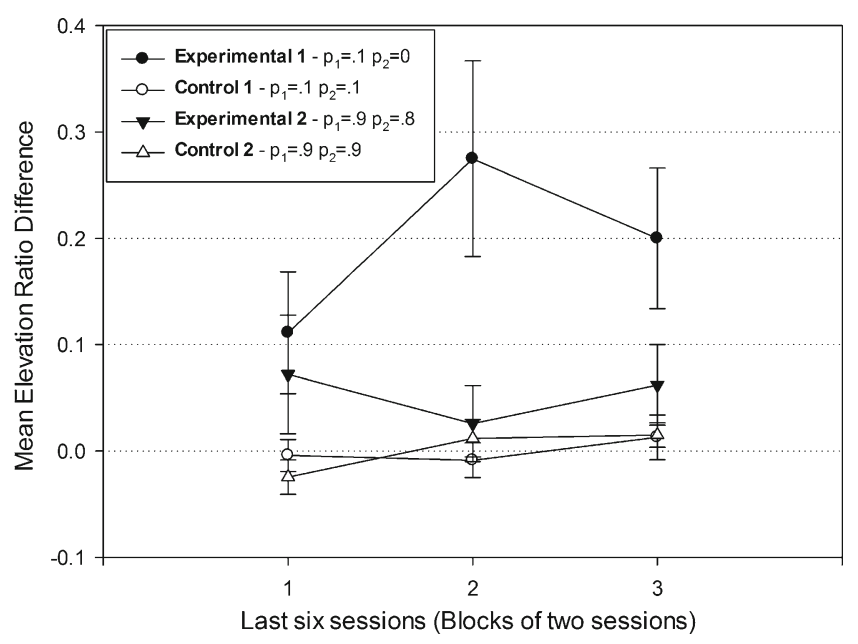

Fig. 7 Mean difference of elevation ratio of CS trials minus the elevation ratio of non-CS trials of the groups of Experiment 4 in the last six conditioning sessions (collapsed in blocks of two). Error bars represent the standard errors of the means

of $\mathrm{p}_{1}$ as compared with its control group with $\Delta \mathrm{p}=0.0$. The same difference was not enough to produce a significant increase of responding in the experimental group, with a high value of $\mathrm{p}_{1}$. As in previous experiments, context-conditioning differences were not found.

\section{General Discussion}

The results of the present experiments have shown that the difference between $p_{1}$ and $p_{2}$ that is required to produce excitatory conditioning in an experimental group, as compared with a control group trained with the same $\mathrm{p}_{1}$ and $\mathrm{p}_{2}$, was not absolute, but that its value increased as the value of $\mathrm{p}_{1}$ increased. Specifically, the experiments have shown that a small difference between $p_{1}$ and $p_{2}(\Delta p=0.1)$ can be enough to produce increased responding to the CS when $\mathrm{p}_{1}$ has a low value, 0.1 (Experiment 1a), but not when $\mathrm{p}_{1}$ has a

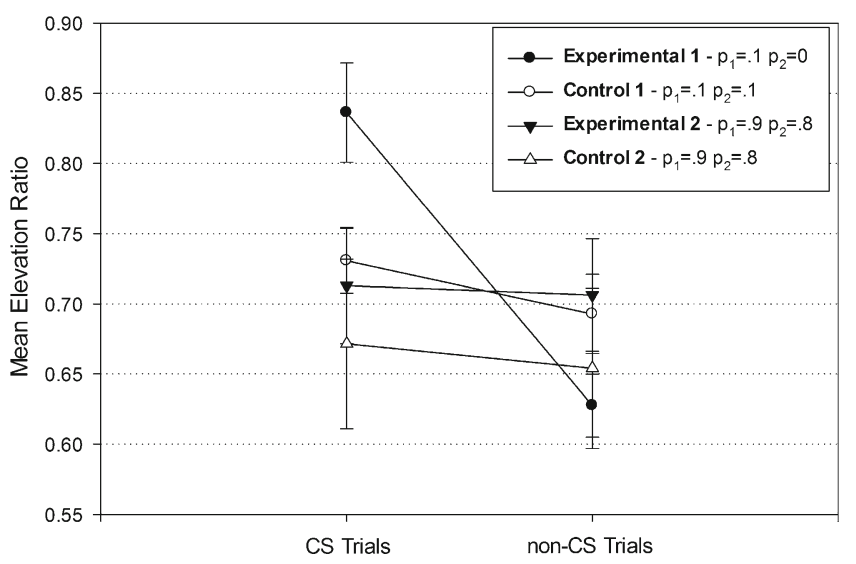

Fig. 8 Mean elevation ratios of CS and non-CS trials of the groups of Experiment 4 in the last six conditioning sessions. Error bars represent the standard errors of the means 
high value, 0.9 (Experiment $1 b$ ). In this case, when $\mathrm{p}_{1}$ is 0.9 , the difference has to be bigger, $\Delta p=0.3$, with this procedure (Experiment 2). When $p_{1}$ has a value of 0.5 , a difference of $\Delta \mathrm{p}=0.2$ was enough to produce excitatory conditioning, whereas a difference of $\Delta \mathrm{p}=0.1$ was not (Experiment 3). The results of Experiments 1a and 1b were replicated in a single experiment (Experiment 4). This result is contrary to the widely accepted idea that whenever $\mathrm{p}_{1}$ is bigger than $\mathrm{p}_{2}$, excitatory conditioning will occur (Rescorla, 1968).

A potential problem of Experiments 1b, 2, and 4 would be the existence of a ceiling effect. Given that a high magnitude of reinforcement was used, it might be possible that a maximum level of responding was reached. This, in turn, could have masked possible differences between high magnitude groups. If this had happened, it might explain to some extent the results found in those experiments, but it cannot explain the whole pattern of results obtained, and therefore does not invalidate the conclusions discussed before. Specifically, in Experiment 3, the probabilities of reinforcement were approximately half of those used in Experiment 1b; the mean response rate of all of the groups of Experiment $1 \mathrm{~b}$ during the CS in the sessions analyzed was of 0.975 responses per second, whereas in Experiment 3, this rate was only $0.722,25 \%$ less. It seems unlikely, therefore, that a ceiling level was reached in both experiments. But in neither experiment was a difference between $p_{1}$ and $p_{2}$ of 0.1 enough to produce an increase in conditioned response with a medium magnitude.

The asymptotic values predicted by some models applied to contingency learning for each particular contingency explored in these experiments are given in Table 3. Six different representative models were used. They are Rescorla and Wagner's (1972) model and $\Delta \mathrm{p}$ (already explained); an adaptation of the Weber-Fechner rule (see below for an extended discussion); and three additional correlational models, $\Delta \mathrm{D}$ (White, 2003), PowerPC (Cheng, 1997), and $\boldsymbol{\Phi}$ (Hammond \& Paynter, 1983). The algebraic expressions of those models not described before can be found in Table 4. As is true for Delta $\Delta \mathrm{p}$, these three models are correlational and are usually described as rule-based models because they do not incorporate a trial $\times$ trial learning mechanism. Instead, they calculate their contingency index on the basis of the frequency of trials and the probabilities derived from them (see Table 4), each one proposing a different contingency rule.

The experimental results that were obtained are incompatible with rule-based models such as $\Delta \mathrm{p}, \Delta \mathrm{D}, \mathrm{PCi}$ (White, 2003), and others (see Allan, 1993, for a review in humans and Hammond \& Paynter, 1983, for nonhumans), but can be explained by the Rescorla and Wagner (1972) model, as long as the value of the parameter $\beta_{1}$ is higher than the value of the parameter $\beta_{2}$. For instance, a simple inspection of Table 3 shows that $\Delta$ p predicts the same level of conditioning in the group with $\mathrm{p}_{1}=0.1$ and $\mathrm{p}_{2}=0$, as in the group with $\mathrm{p}_{1}=0.9$ and $\mathrm{p}_{2}=0.8$. However, Experiments $1 \mathrm{a}, 1 \mathrm{~b}, 2$, and 4 revealed a greater increase of excitatory conditioning in the former group than in the latter, as compared with their respective controls. Similarly, $\Delta \mathrm{D}$ predicts a similar level of learning in a group with $\mathrm{p}_{1}=0.5$ and $\mathrm{p}_{2}=$ 0.3 , and another in which $p_{1}$ is 0.9 and $p_{2}$ is 0.7 . The first group showed significant greater levels of response than its control group, whereas the second did not (Experiments 2 and 3). Something similar happens with PowerPC and $\Phi$ predictions about these groups. Only the Rescorla and Wagner model, when $\beta_{1}$ is higher than $\beta_{2}$, accommodates the results of these experiments.

The effect shown in these experiments is compatible with the density effect, but it cannot be explained by this effect. The density effect (Allan, 1993) is an increase in CR due to an increase in the general probability of appearance of the outcome, but it does not imply a relative difference in discrepancy between $p_{1}$ and $p_{2}$. Density effect has been explained in terms of context conditioning, which could be present in present experiments, but its contribution to the kind of modulation reported here, if any, has been controlled by using elevation ratio as a measure of CR. What is more, no differences in contextual conditioning were found between groups in any of the experiments. In Experiments 1 to 3, direct responses to the context, in absence of the CS, were recorded. This might have reduced the sensitivity of this measure as compared with the CS conditioned response measure, which was an elevation ratio that takes into account the general level of activity of the animal and controls for it. In order to have the same sensitivity in both cases, in Experiment 4, we used a procedure in which contextual conditioning could be measured using an elevation ratio, making its measure equivalent to that of the CS. Even under these conditions, no difference in context conditioning was found between groups that should have differed according to the predictions of Rescorla and Wagner's (1972) model and the rest of the models. Although this finding was not the main objective of the present study, and taking into account that additional experiments focused mainly on exploring it with different procedures and measures are necessary, so far it seems that Rescorla and Wagner's model explains only partially the data obtained.

Another way to conceptualize contingency learning is as a discrimination between $\mathrm{p}_{1}$ and $\mathrm{p}_{2}$. When this discrimination is solved, and $\mathrm{p}_{1}$ is perceived as higher than $\mathrm{p}_{2}$, subjects would show an increase in responding as compared with a situation in which $p_{1}$ and $p_{2}$ are perceived as equal. Using this simple idea as theoretical framework, it is possible to analyze contingency learning in terms of theories and models that come from perception and psychophysics. This would allow us to look for some effects that are commonly found in psychophysics in contingency learning. For instance, the effect that has been described in the present experiments could 
Table 3 Empirical results and simulated predictions of contingency learning models

\begin{tabular}{|c|c|c|c|c|c|c|}
\hline & $0.1-0.0$ & $0.5-0.3$ & $0.5-0.4$ & $0.9-0.6$ & $0.9-0.7$ & $0.9-0.8$ \\
\hline Results & Yes & Yes & No & Yes & No & No \\
\hline$\Delta \mathrm{P}$ & 0.1 & 0.2 & 0.1 & 0.3 & 0.2 & 0.1 \\
\hline$\Delta \mathrm{D}$ & 0.1 & 0.4 & 0.2 & 0.6 & 0.4 & 0.2 \\
\hline$\Phi$ & 0.05 & 0.1 & 0.04 & 0.12 & 0.6 & 0.2 \\
\hline Power PC & 0.11 & 0.29 & 0.17 & 0.75 & 0.67 & 0.5 \\
\hline $\operatorname{RW}\left(\beta_{1} / \beta_{2}=1\right)$ & 0.10 & 0.20 & 0.10 & 0.30 & 0.20 & 0.10 \\
\hline $\mathrm{RW}\left(\beta_{1} / \beta_{2}=0.5\right)$ & 0.05 & 0.16 & 0.08 & 0.39 & 0.28 & 0.15 \\
\hline $\mathrm{RW}\left(\beta_{1} / \beta_{2}=2\right)$ & 0.18 & 0.21 & 0.10 & 0.20 & 0.12 & 0.06 \\
\hline Weber-Fechner & 0.10 & 0.20 & 0.20 & 0.30 & 0.30 & 0.30 \\
\hline
\end{tabular}

The first row indicates the occurrence or not of a significant increase of CR in that group in the results of the experiments. The last row, whose values are in italics, represents the Weber-Fechner rule - that is, the difference needed for the occurrence of differences in the conditioned response given the $\mathrm{p}_{1}$ level in the corresponding column. The intermediate rows symbolize the asymptotic values predicted by rules models and Rescorla and Wagner (1972) assigning three different values to $\beta_{1}$ and $\beta_{2}$

be explained in terms of the Weber-Fechner law, a formal descriptive rule for discrimination and perception. According to this rule, the difference necessary to perceive two stimuli as different $(\Delta S)$ is proportional to the magnitude of the stimuli (S) and is usually a constant $(\mathrm{k})$, specific for each sensory modality. The generalized Weber-Fechner law (Laming, 1986) is expressed as $\Delta S=(S+b) k$ (it includes an additional parameter, $b$, to avoid numerical confusions between increments and absolute values of the stimuli). This generalized law is normally employed when the scale of the absolute values of the $\mathrm{S}$ are very close to the incremental values, as is the case in our experiments. Thus, the effects reported presently can be understood if we adopt a Weber-Fechner modulation of perception in contingency. Indeed, if contingency perception were similar to other perceptual tasks, it would be expected that the bigger the magnitude of the stimulus (in our case: the magnitude of the probability of the US in the presence of the CS), the bigger the difference necessary to perceive as different a new stimulus (in our case, to perceive as different the probability of the US in the absence of the CS). Other features of conditioning have been described using this law; they appear to be perceived according to a logarithmic function. For example, timing seems to follow the WeberFechner law; the scalar property of timing (Gibbon, 1991)

Table 4 Asymptotic algorithms used to generate the data in Table 3

\begin{tabular}{lll}
\hline Model & Asymptotic algorithm & Reference \\
\hline$\Delta \mathrm{p}$ & $p_{1}-p_{2}$ & Jenkins and Ward, 1965 \\
$\Delta \mathrm{D}$ & {$\left[p_{1}+\left(1-p_{2}\right)\right]-\left[p_{2}+\left(1-p_{1}\right)\right]$} & White, 2003 \\
PowerPC & $\left(p_{1}-p_{2}\right) / 1-p_{2}$ & Cheng, 1997 \\
$\Phi$ & $\sqrt{\left(p_{1}-p_{2}\right)\left(p_{3}-p_{4}\right)}$ & Hammond and \\
& & Paynter, 1983 \\
\hline
\end{tabular}

means that longer times are perceived with greater variance than shorter times, and this variance is proportional to the duration being timed.

In fact, the generalized Weber-Fechner law accommodates all of the results described. The results of Experiments 1a and $1 \mathrm{~b}$ showed that a difference between $\mathrm{p}_{1}$ and $\mathrm{p}_{2}$ of 0.1 produces more excitatory conditioning than a control when $\mathrm{p}_{1}$ is 0.1 , but not when $\mathrm{p}_{1}$ is 0.9 . When $\mathrm{p}_{1}$ is 0.9 , that difference has to be of 0.3, according to the results of Experiment 2. Given these values, the parameters of the generalized Weber-Fechner law (Laming, 1986) can be calculated (see Appendix 2). This calculation leads to an estimation of parameters $b$ having a value of 0.3 and $k$ being 0.25 . It is then possible to determine what difference between $\mathrm{p}_{1}$ and $\mathrm{p}_{2}$, according to the model, will be high enough to produce an increase in conditioning $(\Delta S)$ for a given value of $p_{1}(S)$. For example, when $p_{1}=0.5$, the model predicts that only those contingencies with $\Delta \mathrm{p}$ equal or higher than 0.2 will produce excitatory conditioning. The results of Experiment 3 are consistent with the predictions of this rule.

This psychophysical interpretation allows linking two different fields in experimental psychology: learning and perception. This can lead to new ideas and ways to analyze learning situations, and some authors have done this in the past for other aspects of learning (e.g., Allan, Hannah, Crump, \& Siegel, 2008; Boneau \& Cole, 1967; Hack, 1963; Hirsch, 1979; Mason et al., 2003; Nevin, 1964; Schmajuk, 1987). This research has made use of classical psychophysics to deal with a paradigmatic learning issue (contingency). Allan et al. (2008) proposed a psychophysical account of contingency data in which they made use of an account of contingency learning in human subjects based on signal detection theory. Both research programs share a similar conceptual framework based on the usefulness of putting together theories from apparently independent fields such as perception and learning. 
Finally, it is hard to overstate the influence that Rescorla and Wagner's (1972) model has had in associative learning. Both its theoretical aspects and mathematical formalization have been useful tools for researchers for 40 years now. The fact that novel predictions of this model are still being found and tested is a good proof of its longevity and strong predictive validity. Although it predicted correctly only part of the results, it is a powerful research tool and heuristic. Mathematical models of learning are essential research tools under constant development (see, e.g., Le Pelley, 2004; Stout \& Miller, 2007; van Hamme \& Wasserman, 1994; Wagner \& Brandon, 2001)

Author note The present research was supported by a FICYT predoctoral scholarship awarded to S. C., Ficyt/BP07-020. Thanks to Beatriz Álvarez Díaz for her kind help.

\section{Appendix 1}

A MATLAB function was programmed to simulate the data presented in Fig. 1 and supplemental data. It implemented Rescorla and Wagner's (1972) model. The parameters used are presented below.

For the contingency learning simulation, the structure of the training used later in Experiments 1 to 3 was used. Each CS trial was followed by nine trials in which it was absent. The total number of trials was 2,200 in each replica. Each CS trial had a probability of having a US presentation of $\mathrm{p}_{1}$, and, each non-CS trial, of $\mathrm{p}_{2}$. For each condition 10 thousand replicas were run and the results averaged. The value plotted in Fig. 1 is the associative strength of the CS and the context after the last trial of the simulation. Parameter $\alpha$ of the CS was 0.2 . Parameter $\alpha$ of the context was 0.1 . Parameters $\lambda_{1}$ and $\lambda_{2}$ were 1 and 0 , respectively. $\beta_{1}$ and $\beta_{2}$ were 0.2 and 0.1 , respectively. An additional simulation using a wider range of values can be found as supplemental data.

The same function was used to simulate the data of Fig. 6 with a different training structure, in which 30 $\mathrm{CS}$ and 30 non-CS trials were ordered randomly. In each trial, the contextual stimulus was present. Each CS trial had a probability of having a US presentation of $\mathrm{p}_{1}$, and, each non-CS trial, of $\mathrm{p}_{2}$. For each condition, 10 thousand replicas were run and the results averaged. The value plotted in Fig. 6 is the associative strength of the CS and the contextual stimulus after the last trial of the simulation. Parameter $\alpha$ of the CS was 0.2 . Parameter $\alpha$ of the contextual cue was 0.2 . Parameters $\lambda_{1}$ and $\lambda_{2}$ were 1 and 0 , respectively. $\beta_{1}$ and $\beta_{2}$ were 0.2 and 0.1 , respectively.

\section{Appendix 2}

In Appendix 2, we include the calculations of parameters $b$ and $\mathrm{k}$ of the generalized Weber-Fechner law (Laming, 1986) using the results of Experiments 1 and 2. In Experiment 1, we showed that when $p_{1}=0.1$, a difference $\Delta p=0.1$ was enough to observe excitatory conditioning. In Experiment 2, a difference $\Delta \mathrm{p}=0.3$ produced excitatory conditioning when $\mathrm{p}_{1}=$ 0.9 . Given these two points $\left(p_{1}=0.1\right.$ and $\left.p_{1}=0.9\right)$ for which the difference needed to produce excitatory conditioning is known $(\Delta \mathrm{p}=0.1$ and $\Delta \mathrm{p}=0.3$, respectively) and the equation of the generalized Weber-Fechner Law, $\Delta \mathrm{S}=(\mathrm{S}+\mathrm{b}) * \mathrm{k}$,

For $\mathrm{p}_{1}=0.1$ :

$$
\begin{gathered}
0.1=(0.1+\mathrm{b}) * \mathrm{k} \\
\text { For } \mathrm{p}_{1}=0.9:
\end{gathered}
$$

$0.3=(0.9+\mathrm{b}) * \mathrm{k}$

$0.1=0.1 \mathrm{k}+\mathrm{bk}$

$0.3=0.9 \mathrm{k}+\mathrm{bk}$

$0.2=0.8 \mathrm{k}$ then $\mathbf{k}=\mathbf{0 . 2 5}$

and

$0.3=(0.9 * 0.25)+0.25 b$ then $\mathbf{b}=\mathbf{0 . 3}$

\section{References}

Allan, L. G. (1980). A note on measurement of contingency between two binary variables in judgment tasks. Bulletin of the Psychonomic Society, 15, 147-149.

Allan, L. G. (1993). Human contingency judgements: Rule based or associative? Psychological Bulletin, 114, 435-448. doi:10.103700332909.114.3.435

Allan, L. G., Hannah, S. D., Crump, M. J. C., \& Siegel, S. (2008). Psychophysics of Contingency Assessment. Journal of Experimental Psychology. General, 137, 226-243. doi:10.1037//00963445.137.2.226

Baker, A. G., Murphy, R. A., Vallee-Tourangeau, F., \& Mehta, R. (2001). Contingency learning and causal reasoning. In R. R. Mowrer \& S. B. Klein (Eds.), Handbook of contemporary learning theories (pp. 255-306). Mahwah, NJ: Erlbaum.

Boneau, C. A., \& Cole, J. L. (1967). Decision theory, the pigeon, and the psychophysical function. Psychological Review, 74, 123-135. doi: $10.1037 / \mathrm{h} 0024287$

Bueno, M., \& Álvarez, R. (2001). El efecto de las duraciones del intervalo entre ensayos y entre estímulos en el condicionamiento pavloviano apetitivo en ratas. Psicológica, 22, 205-215.

Chapman, G. B., \& Robbins, S. J. (1990). Cue interaction in human contingency judgment. Memory and Cognition, 18, 537-545. doi:10.3758/BF03198486 
Cheng, P. W. (1997). From covariation to causation: A causal power theory. Psychological Review, 104, 367-405. doi:10.1037//0033295X.104.2.367

Crump, M. J. C., Hannah, S. D., Allan, L. G., \& Hord, L. K. (2007). Short article: Contingency judgements on the fly. Quarterly Journal of Experimental Psychology, 60, 753-761. doi:10.1080/ 17470210701257685

Gibbon, J. (1991). Origins of scalar timing. Learning and Motivation, 22, 3-38. doi:10.1016/0023-9690(91)90015-Z

Gluck, M. (1991). Stimulus generalisation and representation in adaptive network models of category learning. Psychological Science, 2, 50-55. doi:10.1111/j.1467-9280.1991.tb00096.x

Hack, M. H. (1963). Signal detection in the rat. Science, 139, 758-759. doi:10.1126/science.139.3556.758

Hammond, L. J., \& Paynter, W. E. (1983). Probabilistic contingency theories of animal conditioning: A critical analysis. Learning and Motivation, 14, 527-550. doi:10.1016/0023-9690(83)90031-

Hirsch, K. A. (1979). Signal detection analysis of conditioning data. The Journal of General Psychology, 101, 249-258. doi:10.1080/ 00221309.1979 .9920078

Holland, P. C. (2000). Trial and intertrial durations in appetitive conditioning in rats. Animal Learning and Behavior, 28, 121-133. doi:10.3758/BF03200248

Jenkins, H. M., \& Ward, W. C. (1965). Judgement of contingency between responses and outcomes. Psychological Monographs: General and Applied, 79, 1-17.

Kamin, L. J. (1969). Predictability, surprise, attention and conditioning. In B. A. Campbell \& R. M. Church (Eds.), Punishment and aversive behavior (pp. 293-294). Oxford, England: AppletonCentury Crofts.

Kirkpatrick, K., \& Church, R. M. (2004). Temporal learning in random control procedures. Journal of Experimental Psychology. Animal Behavior Processes, 30, 213-228.

Laming, D. (1986). Sensory analysis. London, England: Accademic Press. doi:10.1037/0097-7403.30.3.213

Lattal, K. M., \& Nakajima, S. (1998). Overexpectation in appetitive Pavlovian and instrumental conditioning. Animal Learning and Behavior, 26, 351-360. doi:10.3758/BF03199227

Le Pelley, M. E. (2004). The role of associative history in models of associative learning: A selective review and a hybrid model. Quarterly Journal of Experimental Psychology, 57B, 193-243. doi:10.1080/02724990344000141

Luque, D., López, F. J., Marco-Pallares, J., Càmara, E., \& RodríguezFornells, A. (2012). Feedback-related brain potential activity complies with basic assumptions of associative learning theory. Journal of Cognitive Neuroscience, 24, 794-808. doi:10.1162/ jocn_a 00145

Mackintosh, N. J. (1975). A theory of attention: Variations in the associability of stimuli with reinforcement. Psychological Review, 82, 276-298. doi:10.1037/h0076778

Mason, C. R., Idrobo, F., Early, S. J., Abibi, A., Zheng, L., Harrison, J. M., \& Carney, L. H. (2003). CS-dependent response probability in an auditory masked-detection task: Considerations based on models of Pavlovian conditioning. The Quarterly Journal of Experimental Psychology, Section B, 56, 193-205. doi:10.1080/ 02724990244000052
Miller, R. M., Barnet, R. C., \& Grahame, N. J. (1995). Assessment of the Rescorla-Wagner, odel. Psychological Bulletin, 117, 363 386. doi:10.1037/0033-2909.117.3.363

Murphy, R. A., \& Baker, A. G. (2004). A role for CS-US contingency in Pavlovian conditioning. Journal of Experimental Psychology: Animal Behavior Processes, 30, 229-239.

Murphy, R. A., Baker, A. G., \& Fouquet, N. (2001). Relative validity effects with either one or two more valid cues in Pavlovian and instrumental conditioning. Journal of Experimental Psychology. Animal Behavior Processes, 27, 59-67. doi:10.1037/0097-7403.27.1.59

Nevin, J. A. (1964). A method for the determination of psychophysical functions in the rat. Journal of Experimental Analysis of Behavior 7, 169 .

Papini, M. R., \& Bitterman, M. E. (1990). The role of contingency in classical conditioning. Psychological Review, 97, 396-403. doi:10.1037/0033-295X.97.3.396

Pearce, J. M., \& Hall, G. (1980). A model for Pavlovian learning: Variations in the effectiveness of conditioned but not of unconditioned stimuli. Psychological Review, 87, 532-552. doi:10.1037/ 0033-295X.87.6.532

Rescorla, R. A. (1968). Probability of shock in the presence and absence of CS in fear conditioning. Journal of Comparative and Physiological Psychology, 66, 1-5. doi:10.1037/h0025984

Rescorla, R. A. (2004). Superconditioning from a reduced reinforcer. Quarterly Journal of Experimental Psychology, 57B, 133-152. doi:10.1080/02724990344000051

Rescorla, R. A., \& Wagner, A. R. (1972). A theory of Pavlovian conditioning: Variations in the effectiveness of reinforcement and nonreinforcement. In A. H. Black \& W. F. Prokasy (Eds.), Classical conditioning II: Current research and theory (pp. 64-99). New York, NY: Appleton.

Schmajuk, N. A. (1987). Classical conditioning, signal detection and evolution. Behavioural Processes, 14, 277-289. doi:10.1016/ 0376-6357(87)90074-X

Schultz, W. (2002). Getting formal with dopamine and reward. Neuron, 36, 241-263. doi:10.1016/S0896-6273(02)00967-4

Soto, F. A., \& Wasserman, E. A. (2010). Error-driven learning in visual categorization and object recognition: A common elements model. Psychological Review, 117, 349-381. doi:10.1037/a0018695

Stout, S. C., \& Miller, R. R. (2007). Sometimes competing retrieval (SOCR): A formalization of the comparator hypothesis. Psychological Review, 114, 759-783.

Van Hamme, L. J., \& Wasserman, E. A. (1994). Cue competition in causality judgments: The role of nonpresentation of compound stimulus elements. Learning and Motivation, 25, 127-151. doi:10.1006/lmot.1994.1008

Wagner, A. R., \& Brandon, S. E. (2001). A componential theory of Pavlovian conditioning. In R. R. Mower \& S. B. Klein (Eds.), Handbook of contemporary learning theories (pp. 23-64). Mahwah, NJ: Erlbaum

Wagner, A. R., Logan, F. A., Haberlandt, K., \& Price, T. (1968). Stimulus selection in animal discrimination learning. Journal of Experimental Psychology, 76, 171-180. doi:10.1037/h0025414

White, P. A. (2003). Making causal judgments from the proportion of confirming instances: The pCI rule. Journal of Experimental Psychology: Learning, Memory, and Cognition, 29, 710-727. doi:10.1037/0278-7393.29.4.71 